\title{
Exposure to cooking oil fumes and chronic bronchitis in nonsmoking women aged 40 years and over: a health-care based study
}

Huang-Chi Chen ${ }^{1+}$, Chia-Fang Wu ${ }^{2+}$, Inn-Wen Chong ${ }^{4,5}$ and Ming-Tsang Wu $\mathbf{u}^{2,3,6^{*}}$

\begin{abstract}
Background: Little is known about the effect of exposure to cooking oil fumes (COFs) on the development of non-malignant respiratory diseases in nonsmoking women. This study investigated the relationship between exposure to COFs and chronic bronchitis in female Taiwanese non-smokers.

Methods: Searching the 1999 claims and registration records maintained by Taiwan's National Health Insurance Program, we identified 1846 women aged 40 years or older diagnosed as having chronic bronchitis (ICD-9 code: 491) at least twice in 1999 as potential study cases and 4624 women who had no diagnosis of chronic bronchitis the same year as potential study controls. We visited randomly selected women from each group in their homes, interviewed to collect related data including cooking habits and kitchen characteristics, and them a spirometry to collect FEV1 and FVC data between 2000 and 2009.

Results: After the exclusion of thirty smokers, the women were classified those with chronic bronchitis $(n=53)$, probable chronic bronchitis $(n=285)$, and no pulmonary disease $(n=306)$ based on physician diagnosis and American Thoracic Society criteria. Women who had cooked $\geq 21$ times per week between the ages of 20 and 40 years old had a 4.73-fold higher risk of chronic bronchitis than those cooking $<14$ times per week $(95 \% \mathrm{Cl}=1.65-13.53)$. Perceived kitchen smokiness was significantly associated with decreased FEV1 $(-137 \mathrm{ml}, p=0.021)$ and FEV1/FVC ratio $(-7.67 \%, p=0.008)$.
\end{abstract}

Conclusions: Exposure to COF may exacerbate the progression of chronic bronchitis in nonsmoking women.

Keywords: Chronic bronchitis, Cooking oil fumes, Pulmonary function test, Nonsmoking women

\section{Background}

Chronic obstructive pulmonary disease (COPD) was ranked by World Health Organization (WHO) as the third leading cause of death in 2012, causing 3.1 million deaths globally and $5.6 \%$ of all deaths worldwide [1]. In Taiwan, the prevalence rate for this disease exceeds $6 \%$ $(>6.1 \%)$ in adults older than 40 years [2]. COPD is potentially irreversible and patients with this disease in

\footnotetext{
* Correspondence: 960021@ms.kmuh.org.tw; e_encourage@yahoo.com ${ }^{\dagger}$ Equal contributors

${ }^{2}$ Department of Public Health, College of Health Sciences, Kaohsiung Medical University, Kaohsiung, Taiwan

${ }^{3}$ Research Center for Environmental Medicine, Kaohsiung Medical University, Kaohsiung, Taiwan

Full list of author information is available at the end of the article
}

Taiwan are estimated to spend more than 1.8 million US dollars per year of medical treatment for this disease [3].

COPD is traditionally more common in males because a much larger proportion of men smoked cigarettes, a known cause of COPD. However, due to increases in tobacco use by women in high-income countries and increased risk of indoor air pollution in low-income countries, that gap is beginning to close [4]. Still cigarette smoking is much less prevalent in women in Taiwan than in Caucasian women in other countries (3-4\% vs. 28\%) $[5,6]$. Thus, there may other factors besides smoking related to the development of COPD in Taiwanese women. One of our previous healthcare based studies, for example, found women exposed to second-hand smoke (SHS) were 
3.65-fold more likely to have chronic bronchitis than nonexposed women in Taiwan [7], suggesting the possibility of other lifestyle or airborne factors.

Taiwanese women are customarily responsible for preparing the meals for their families. Taiwanese-style cooking often involves pre-heating oil to smoking point before adding the ingredients for stir-frying or deepfrying, most often over natural gas or electric burners. Previous studies have found several carcinogens, including polycyclic aromatic hydrocarbons (PAHs), aromatic amines, and nitro-polycyclic aromatic hydrocarbons, in cooking oil fumes (COFs) and in the kitchens of Chinese homes where women prepare food daily [8-11]. In addition to carcinogens, Taiwanese-style cooking can generate other irritating chemicals, such as 1,3-butadiene, formaldehyde, and aldehyde [12-14] which can affect the respiratory tract and lead to inflammation of the airway. Although exposure to COFs has been associated with risk for several cancers, including lung and cervix [15-19], its relationship to chronic non-cancerous respiratory diseases, such as chronic bronchitis, remains unclear. Thus, we sought to extend our previous healthcare study beyond effect of second-hand smoke and chronic bronchitis, this time focusing on COF. To do this, we tapped Taiwan's 1999 National Health Insurance (NHI) Database and NHI Registration Database to identify women forty years old who had been residents of Kaohsiung City, Taiwan, for five years or more and who had a previous diagnosis of chronic bronchitis or probable chronic bronchitis and the same population of women who had not been diagnosed with this disease. The women were visited in their homes and administered a survey which included questions about cooking habits and kitchen characteristics and gave them a spirometry test to collect lung function data (FEV1 and FVC).

\section{Methods}

\section{Study area and study population}

This ongoing healthcare based study has been described in detail previously [7]. Briefly, the 1999 claims and registration records of women aged 40 years or older living in Kaohsiung City for five years or more were identified in the database of Taiwan's Kao-Ping District National Health Insurance Bureau, a branch of Taiwan's National Health Insurance Program [20]. Kaohsiung city is a heavily industrialized harbor city located on the southwestern coast of Taiwan $\left(153.6 \mathrm{~km}^{2}\right)$ (Additional file 1: Figure S1).

A total of 221,965 women 40 years of age or older had lived in the city for five or more years and had made health insurance claims in 1999 [7]. Those diagnosed as having chronic bronchitis (ICD-9 code: 491) at least twice in 1999 were considered potential members of our study group (cases). Those who had made physician visits for traffic accidents (ICD-9 code: E800-E848) or acute gastroenteritis (ICD-9 code: 008.8; 009.1; 558.3; 558.9) the same year and who had never been diagnosed as having chronic bronchitis were considered potential study controls. Any case or control subject was excluded she had been diagnosed with other pulmonaryassociated diseases, including asthma (ICD-9 code: 493), pulmonary tuberculosis (ICD-9 code: 011.9 or 010-018), bronchiectasis (ICD-9 code: 494), fibrotic cyst (ICD-9 code: 277), pulmonary tumor (ICD-9 code:162), emphysema (ICD-9 code: 492), extrinsic allergic alveolitis (ICD-9 code: 495), or chronic airway obstruction, not elsewhere classified (ICD-9 code: 496). After exclusion, we were left with 1846 potential study cases and 4624 potential controls (Fig. 1).

We randomly selected about one-third of 1846 potential study cases $(n=622)$ for subsequent in-person interviews and pulmonary function tests in their homes and randomly selected 1244 of the 4624 controls matching potential cases by age (within 3 years) and administrative area (Fig. 1). Once a study case was successfully recruited, interviewed and administered a pulmonary function test, one matched control was also immediately recruited, interviewed, and tested to adjust for the possible influence from external environmental hazards such as air pollution from large factories or traffic. In total, 337 cases and 337 controls completed the interview and pulmonary function tests between June 2000 and March 2009. This study was approved by IRB at Kaohsiung Medical University. All participants provided signed informed consent.

\section{Measurement of COF exposure Questionnaire}

Trained interviewers conducted personal face-to-face home interviews with the participants to collect epidemiologic data using a standardized questionnaire modified from the American Thoracic Society Division of Lung Disease Respiratory Symptom Questionnaire [2]. It included questions to collect demographic data including age, gender, educational level, ethnicity, and marital status as well as participant characteristics including family history of respiratory diseases, environmental chemical exposures in homes (second-hand smoke, COFs, mosquito coil burning and incense burning), and symptoms of chronic bronchitis.

We based our questions related to COF exposure on those we used in a previous community study of etiology of cervical intraepithelial neoplasm [19, 21]. Each participant was asked whether she cooked at home at least once a week between the ages of 20 and 40 years old. This question was also used in a study of Chinese food cooking and lung cancer risk in Taiwanese women [5]. The age range in this question is based on the assumption that Taiwanese women generally start cooking at the time of 


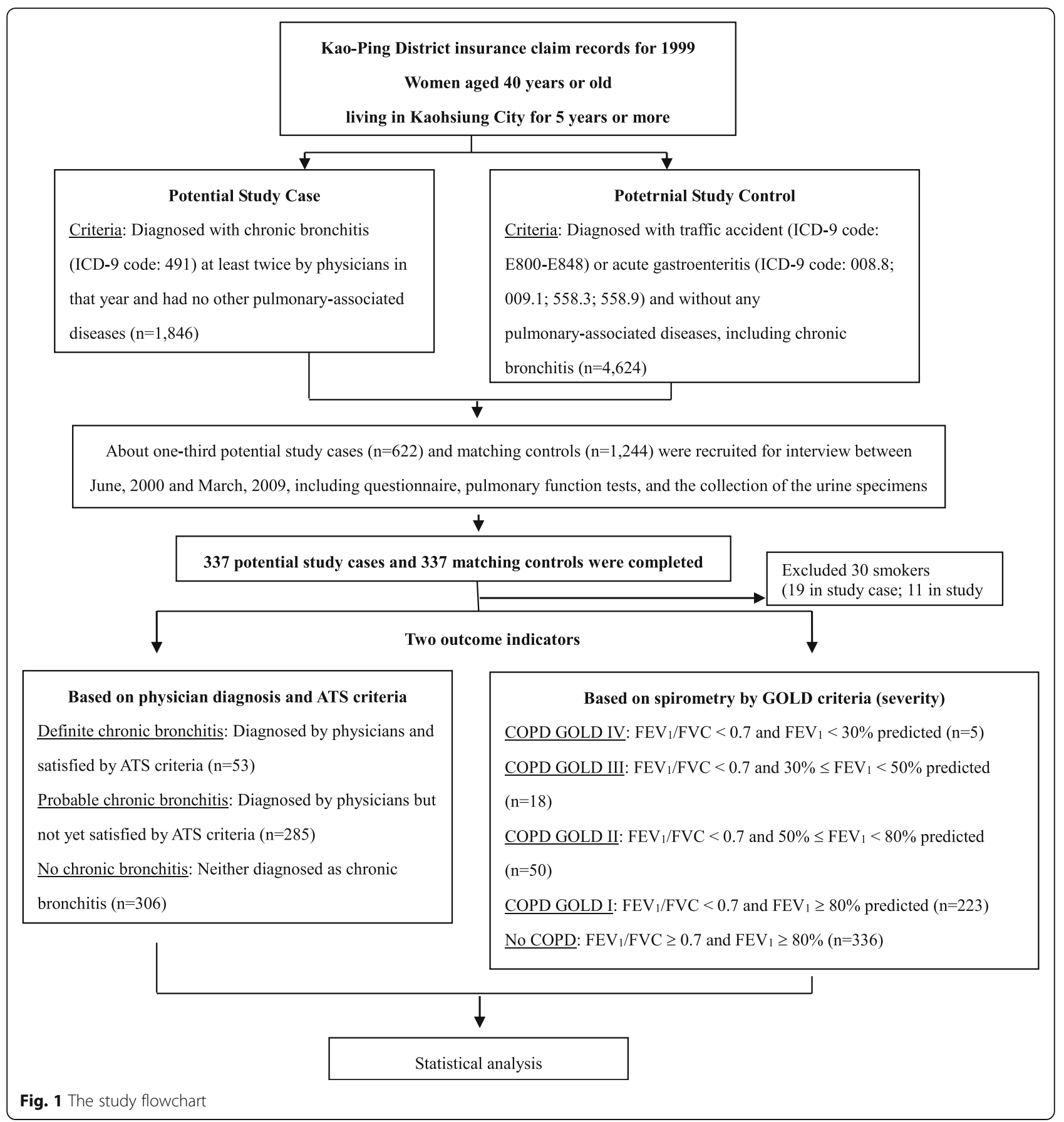

marriage (around 20 years old) and quit cooking at around the age of 40 years when their daughters-in-laws traditionally take over their duties. The daughter-in-law of the first son often moves into the house of her in-laws in an extended family in Taiwan. The age range in this question also takes into consideration the latent period between the time of exposure to $\mathrm{COF}$ and the occurrence of chronic diseases, such as COPD [19].

If a participant answered that she had cooked at home at least once a week, she was asked a detailed set of questions on cooking and ventilation conditions. These questions covered when she started cooking at home, how many years she had cooked, how many hours on average per day she stayed in the kitchen, what kind of fuels she used, whether there was a kitchen fume extractor installed in the home kitchen or not, how often the extractor was turned on while cooking, and whether she perceived the kitchen to be smoky or experienced any eye irritation while cooking. The interview lasted no more than $30 \mathrm{~min}$ on average. The interviewers could 
not be blinded to the health status of potential case/control. However, they only knew this activity was one part of health-promotion program and did not know the main hypothesis of this study.

\section{Reliability of COF questions}

One of our previous studies evaluated the reproducibility of the COF question items and found the test-retest reliabilities for most questions to have Kappa statistics (k) coefficients ranging between 0.69 and 0.75 , indicating moderate to substantial agreement in one hundred study women [21]. In the current study, one to three months after the first interview, we re-asked 78 participants the question, "Between 20 and 40 years old, did you regularly cook at home (regularly, defined as at least once a week)?" The k coefficient was $0.51(95 \% \mathrm{CI}=0.33-0.75)$, suggesting moderate agreement [22].

\section{Outcome measurement}

\section{Pulmonary function test}

After each interview, each participant was administered a pulmonary function test using a portable spirometer (Micro Direct Inc. MSO3, Rochester, England). The measurement included forced expiratory volume in 1st second $\left(\mathrm{FEV}_{1}\right)$ and forced vital capacity (FVC) [2]. Each participant performed the pulmonary function test three times and the highest values were recorded. None of the participants had smoked, eaten, or used any bronchodilators one hour before performing the pulmonary function test. All pulmonary function tests were administered following American Thoracic Society (ATS) guidelines [23]. To calculate the percent predicted values for lung function, we used predictive equations derived from Baldwin's formula for FVC and Berglund's formula for FEV1 [24, 25].

\section{Chroni'c bronchitis related health status}

We used two disease classification systems to categorize chronic bronchitis-related health status in our data analysis, as described in detail previously [7]. First, we classified all study subjects into three disease groups based on a physician's diagnosis of chronic bronchitis at least twice in year 1999 and on ATS criteria for chronic bronchitis (the presence of cough and/or sputum production during the majority of days for at least three consecutive months in the previous two or more successive years) [23]. Subjects fulfilling both requirements were categorized as having "definite chronic bronchitis", fulfilling one as having "probable chronic bronchitis", and not fulfilling either as having "no pulmonary disease". Second, using pulmonary function test data (FEV1 and FVC), we categorized COPD into various levels of severity as described previously using National Heart, Lung, and Blood Institute/World Health Organization GOLD criteria (Stages 0-IV) [26]: Stage 0, normal lung function
$\left(\mathrm{FEV}_{1} / \mathrm{FVC} \geq 70 \%\right.$ and $\mathrm{FEV}_{1} \geq 80 \%$ predicted); Stage I, airflow mildly limited $\left(\mathrm{FEV}_{1} / \mathrm{FVC}<70 \%\right.$ and $\mathrm{FEV}_{1} \geq 80 \%$ predicted); Stage II, airflow increasingly limited $\left(\mathrm{FEV}_{1} /\right.$ $\mathrm{FVC}<70 \%$ and $50 \% \leq \mathrm{FEV}_{1}<80 \%$ predicted); Stage III $\left(\mathrm{FEV}_{1} / \mathrm{FVC}<70 \%\right.$ and $30 \% \leq \mathrm{FEV}_{1}<50 \%$ predicted) and finally Stage IV $\left(\mathrm{FEV}_{1} / \mathrm{FVC}<70 \%\right.$ and $\mathrm{FEV}_{1}<30 \%$ predicted), severe airflow limitation. For this study, Stage 0 was grouped as "No COPD" and Stage I as "Mild COPD". Because the sample size was small, Stages II-IV were categorized as "Moderate COPD" (Fig. 1).

\section{Statistical analysis}

According to our previous published results [7], case and control response rates were $67.3 \%$ and $68.4 \%$, respectively. The average age $( \pm S D)$ (years) of case responders and non-responders were not significantly different ( $64.6 \pm 9.6$ vs. $63.2 \pm 10.8, p=0.11)$, and neither were age of the control responders and non-responders $(64.6 \pm 9.7$ vs. $64.7 \pm 11.2 ; p=0.91)$. Because smoking is the main factor leading to chronic bronchitis [7], we excluded 19 smokers in the cases and 11 in the controls. Group characteristics were analyzed by the two disease classification systems and tested $\chi^{2}$ and Fisher's exact tests, or ANOVA statistics when appropriate.

Polytomous logistic regressions were used evaluate the association between different COF variables and health status outcomes, adjusting for age, height, education (illiteracy vs. $\geq$ junior high school; primary school vs. $\geq$ junior high school), smoking status (SHS vs. nonsmoker), incense burning (yes vs. no), and tea consumption (yes vs. no), all the potential covariates related to the risk of COPD, as suggested by our previous study [7]. Multiple linear regressions were used to investigate the relationship between the same individual variables and pulmonary function, either $\mathrm{FEV}_{1}$ or FEV1/FVC, both normally distributed. All statistical operations were performed using the SAS statistical package. Odds ratio (OR) and 95\% confidence interval (CI) were calculated, and all $p$-values were two-sided.

\section{Results}

After excluding thirty smokers (19 potential study cases and 11 controls), we reclassified the participants into three groups: "definite chronic bronchitis" $(n=53)$, "probable chronic bronchitis" $(n=285)$, and "no pulmonary disease" $(n=306)$ (Table 1; Additional file 1: Table S1). While none of the 326 controls had definite chronic bronchitis, twenty (6.1\%) were classified as having probable chronic bronchitis (Additional file 1: Table S2). FEV1, smoking status, and tea consumption were significantly different across the groups before and after reclassification.

As can be seen in Table 2, which analyzes the relationships between different COF exposure variables and risk of chronic bronchitis among non-smoking women, most 
Table 1 Participant characteristics and pulmonary function test results of 644 non-smoking women categorized by physician diagnosed and ATS criteria

\begin{tabular}{|c|c|c|c|c|}
\hline Variables & $\begin{array}{l}\text { Definite chronic } \\
\text { bronchitis }(N=53)\end{array}$ & $\begin{array}{l}\text { Probably chronic } \\
\text { bronchitis }(N=285)\end{array}$ & $\begin{array}{l}\text { No pulmonary } \\
\text { disease }(N=306)\end{array}$ & $p$-value \\
\hline \multicolumn{5}{|l|}{ Mean \pm SD or N (\%) } \\
\hline Age (yrs) & $61.9 \pm 9.4$ & $63.3 \pm 8.8$ & $63.3 \pm 9.2$ & 0.558 \\
\hline Height (cm) & $155.1 \pm 6.1$ & $155.6 \pm 5.7$ & $155.1 \pm 5.4$ & 0.494 \\
\hline Weight (kg) & $57.3 \pm 13.0$ & $58.6 \pm 8.6$ & $59.1 \pm 8.6$ & 0.392 \\
\hline BMl & $23.8 \pm 5.4$ & $24.2 \pm 3.3$ & $24.6 \pm 3.4$ & 0.216 \\
\hline \multicolumn{5}{|l|}{ Pulmonary function test } \\
\hline $\mathrm{FEV}_{1}(\mathrm{~L})^{\mathrm{a}}$ & $1.47 \pm 0.45$ & $1.53 \pm 0.44$ & $1.62 \pm 0.47$ & 0.012 \\
\hline $\mathrm{FVC}(\mathrm{L})^{\mathrm{a}}$ & $1.85 \pm 0.55$ & $1.91 \pm 0.55$ & $1.92 \pm 0.58$ & 0.720 \\
\hline $\mathrm{FEV}_{1} / \mathrm{FVC}(\%)^{\mathrm{a}}$ & $80.5 \pm 13.5$ & $81.0 \pm 14.7$ & $86.0 \pm 14.4$ & $<0.0001$ \\
\hline \multicolumn{5}{|l|}{ Education levels ${ }^{\mathrm{b}}$} \\
\hline$\geq$ junior high school & $10(18.9)$ & $52(18.3)$ & $44(14.4)$ & 0.343 \\
\hline primary school & $25(47.2)$ & $138(48.4)$ & $172(56.4)$ & \\
\hline illiteracy & $18(34.0)$ & 95 (33.3) & $89(29.2)$ & \\
\hline \multicolumn{5}{|l|}{ Smoking status } \\
\hline Non-smoker & $4(7.6)$ & $79(27.7)$ & $106(34.6)$ & 0.0002 \\
\hline Second-hand smoker & $49(92.5)$ & $206(72.3)$ & $200(65.4)$ & \\
\hline \multicolumn{5}{|l|}{ Tea consumption } \\
\hline No & $50(94.3)$ & $241(84.6)$ & $236(77.1)$ & 0.003 \\
\hline Yes & $3(5.7)$ & $44(15.4)$ & $70(22.9)$ & \\
\hline \multicolumn{5}{|l|}{ Burning incense } \\
\hline No & $15(28.3)$ & $112(39.3)$ & $120(39.2)$ & 0.291 \\
\hline Yes & $38(71.7)$ & $173(60.7)$ & $186(60.8)$ & \\
\hline
\end{tabular}

ATS American Thoracic Society; BMI body mass index; FEV1 forced expiratory volume in 1st second; FVC forced vital capacity; SD standard deviation ${ }^{\mathrm{a}}$ Missing data $=7(3,3,1)$

${ }^{\mathrm{b}}$ Missing data $=1(0,0,1)$

of the participants cooked at least once a week between the ages 20 to 40 years $(87.8 \%$ of those with no pulmonary disease and $>92 \%$ of those with probable and definite chronic bronchitis). The median age at which the women started cooking in a home kitchen was 22 years old. The greater the number of meals cooked per week, the higher the risk probable and definite chronic bronchitis, after adjusting for other covariates, including age, SHS status, height, education level, burning incense, and tea consumption. The risk for definite chronic bronchitis was highest in women who cooked $\geq 21$ meals per week (adjusted $\mathrm{OR}=4.73 ; \quad 95 \% \quad \mathrm{CI}=1.65-13.53$ ) (Table 2; Additional file 1: Table S3).

Applying the GOLD criteria, we found kitchen smokiness and eye irritation while cooking to be significantly associated with risk of moderate COPD and mild + moderate COPD, respectively (Table 3; Additional file 1: Table S4). Those who had cooked $\geq 21$ meals per week were at relatively higher risk of mild or moderate COPD than those who had cooked $<14$ meals per week, though significance was not reached (Table 3; Additional file 1: Table S4). With regard to pulmonary function, number of meals cooked per week and kitchen smokiness were negatively and significantly associated with FEV1 and FEV1/FVC ratio after adjustment (Table 4). For example, kitchen smokiness was significantly associated with the decreased FEV1 $(-137 \mathrm{ml}, p=0.021)$ and FEV1/FVC ratio $(-7.67 \%, p=0.008)$ in non-smoking Taiwanese women. Good kitchen ventilation was significantly associated with higher FEV1/FVC ratio, but not FEV1.

\section{Discussion}

This study found that the frequency of meals cooked per week and kitchen smokiness while cooking between the ages of 20- and 40-years-old to be the main COF determinants for progression of later chronic bronchitis and impaired lung function in non-smoking women in Taiwan and that good kitchen ventilation may reduce impairment of lung function. 
Table 2 Relationships between variables of cooking oil fume exposure and the risk of chronic bronchitis among non-smoking women $(N=644)$

\begin{tabular}{|c|c|c|c|c|c|c|c|}
\hline \multirow[t]{2}{*}{ Variables } & \multirow{2}{*}{$\begin{array}{l}\text { No pulmonary } \\
\text { disease }(N=306) \\
N(\%)\end{array}$} & \multicolumn{3}{|c|}{ Probably chronic bronchitis $(N=285)$} & \multicolumn{3}{|c|}{ Definite chronic bronchitis $(N=53)$} \\
\hline & & $\mathrm{N}(\%)$ & COR $(95 \% \mathrm{Cl})$ & AOR $(95 \% \mathrm{Cl})^{*}$ & $\mathrm{~N}(\%)$ & COR $(95 \% \mathrm{Cl})$ & AOR $(95 \% \mathrm{Cl})^{*}$ \\
\hline \multicolumn{8}{|l|}{ Smoking status } \\
\hline Non-smoker & $106(34.6)$ & $79(27.7)$ & 1 & 1 & $4(7.6)$ & 1 & 1 \\
\hline Second-hand smoker & $200(65.4)$ & $206(72.3)$ & $1.38(0.97-1.96)$ & $1.42(0.99-2.03)$ & $49(92.5)$ & $6.49(2.28-18.48)$ & $6.66(2.31-19.20)$ \\
\hline \multicolumn{8}{|l|}{ Cooked in the kitchen } \\
\hline No & $22(7.2)$ & $13(4.6)$ & 1 & 1 & $5(9.4)$ & 1 & 1 \\
\hline Yes & $284(92.8)$ & $272(95.4)$ & $1.62(0.80-3.82)$ & $1.65(0.80-3.43)$ & $48(90.6)$ & $0.74(0.27-2.06)$ & $0.73(0.22-2.43)$ \\
\hline \multicolumn{8}{|l|}{ Excluding no cook } \\
\hline \multicolumn{8}{|l|}{ Age stared cooking } \\
\hline$>22$ years & $126(44.4)$ & $132(48.5)$ & 1 & 1 & $21(43.8)$ & 1 & 1 \\
\hline$\leq 22$ years & $158(55.6)$ & $140(51.5)$ & $0.85(0.61-1.18)$ & $0.86(0.61-1.23)$ & $27(56.3)$ & $1.03(0.55-1.90)$ & $1.13(0.58-2.20)$ \\
\hline \multicolumn{8}{|l|}{ Meals per week } \\
\hline $1-13$ & $85(29.9)$ & $55(20.2)$ & 1 & 1 & $5(10.4)$ & 1 & 1 \\
\hline $14-20$ & $94(33.1)$ & $84(30.9)$ & $1.38(0.88-2.16)$ & $1.52(0.95-2.42)$ & $14(29.2)$ & $2.53(0.88-7.32)$ & $2.71(0.84-8.14)^{\S}$ \\
\hline $21-31$ & $105(37.0)$ & $133(48.9)$ & $1.96(1.28-2.99)$ & $2.14(1.36-3.36)^{\dagger}$ & $29(60.4)$ & $4.69(1.74-12.65)$ & $4.73(1.65-13.53)^{\dagger}$ \\
\hline \multicolumn{8}{|l|}{ Fuel used for cooking ${ }^{a}$} \\
\hline Gas/electric stove & $179(63.0)$ & $152(56.1)$ & 1 & 1 & $28(58.3)$ & 1 & 1 \\
\hline Biomass fuels & $67(23.6)$ & $68(25.1)$ & $1.20(0.80-1.78)$ & $1.39(0.85-2.26)$ & $12(25.0)$ & $1.15(0.55-2.38)$ & $1.15(0.47-2.85)$ \\
\hline Coal & $38(13.4)$ & $51(18.8)$ & $1.58(0.99-2.54)$ & $1.83(1.07-3.13)^{\ddagger}$ & $8(16.7)$ & $1.35(0.57-3.18)$ & $1.36(0.51-3.65)^{\ddagger}$ \\
\hline \multicolumn{8}{|c|}{ Windows in the home kitchen ${ }^{\text {b }}$} \\
\hline No & $15(5.3)$ & $17(6.4)$ & 1 & 1 & $6(13.6)$ & 1 & 1 \\
\hline$\geq 1$ & $266(94.7)$ & $247(93.6)$ & $0.82(0.40-1.68)$ & $0.80(0.38-1.68)$ & $38(86.4)$ & $0.36(0.13-0.98)$ & $0.40(0.14-1.16)$ \\
\hline \multicolumn{8}{|c|}{ Ventilation in the home kitchen ${ }^{c}$} \\
\hline Poor & $14(5.0)$ & $9(3.3)$ & 1 & 1 & $6(12.5)$ & 1 & 1 \\
\hline Good & $269(95.1)$ & $261(96.7)$ & $1.51(0.64-3.55)$ & $1.46(0.61-3.50)$ & $42(87.5)$ & $0.36(0.13-1.00)$ & $0.35(0.12-1.04)$ \\
\hline \multicolumn{8}{|l|}{ Installed fume extractor ${ }^{d}$} \\
\hline No & $122(43.6)$ & $128(48.3)$ & 1 & 1 & $25(52.1)$ & 1 & 1 \\
\hline Yes & $158(56.4)$ & $137(51.7)$ & $0.83(0.59-1.16)$ & $0.75(0.50-1.12)$ & $23(47.9)$ & $0.71(0.39-1.31)$ & $0.69(0.34-1.43)$ \\
\hline \multicolumn{8}{|c|}{ Eye irritation during cooking ${ }^{e}$} \\
\hline No & $241(96.0)$ & $238(96.0)$ & 1 & 1 & $42(93.3)$ & 1 & 1 \\
\hline Yes & $10(4.0)$ & $10(4.0)$ & $1.01(0.41-2.48)$ & $1.05(0.42-2.62)$ & $3(6.7)$ & $1.72(0.46-6.52)$ & $1.97(0.48-8.01)$ \\
\hline \multicolumn{8}{|c|}{ Smokiness during cooking ${ }^{f}$} \\
\hline No & $232(92.1)$ & $226(91.1)$ & 1 & 1 & $40(88.9)$ & 1 & 1 \\
\hline Yes & $20(7.9)$ & $22(8.9)$ & $1.13(0.60-2.13)$ & $1.17(0.61-2.24)$ & $5(11.1)$ & $1.45(0.52-4.09)$ & $1.36(0.46-4.00)$ \\
\hline
\end{tabular}

AOR adjusted OR; COR crude OR; OR odds ratio

*Adjusting for age, second hand smoke status, height, education level, burning incense, and tea consumption

${ }^{+}$Trend test from 1 to $13,14-20$, to $21-31$ meals per week in probably chronic bronchitis: $p=0.009$; and in chronic bronchitis: $p=0.0023$

${ }^{\ddagger}$ Trend test from gas/electric stove, biomass fuels, to coal in probably chronic bronchitis: $p=0.025$; and in chronic bronchitis: $p=0.544$

${ }^{\S}$ Trend test from no pulmonary disease, probably chronic bronchitis, to chronic bronchitis in 14-20 meals per week: $p=0.020$; and in 21-31 meals per week: $p<0.0001$

${ }^{\mathrm{a}}$ Missing data, $n=1(0,1,0)$. ${ }^{\mathrm{b}}$ Missing data, $n=15(3,8,4)$. ${ }^{\mathrm{c} M i s s i n g}$ data, $n=3(1,2,0)$. ${ }^{\mathrm{d}}$ Missing data, $n=11(4,7,0)$. ${ }^{\mathrm{e}}$ Missing data, $n=60(33,24,3) .{ }^{\mathrm{f}} \mathrm{Missing}$ data,

$n=59(32,24,3)$

COFs contain more than two hundred kinds of harmful particulates and gases, many of which have been identified as human carcinogens and environmental irritants $[8-11,27]$. These chemicals include benzene, formaldehyde, 1,3-butadiene, aromatic amines, polycyclic aromatic hydrocarbons (PAHs) such as benzo[a]pyrene $(\mathrm{B}[\mathrm{a}] \mathrm{P})$, benz $[\mathrm{a}]$ anthracene, and dibenz $[\mathrm{a}, \mathrm{h}]$ anthracene, and acrolein, all known to cause not only mutagenicity 
Table 3 Relationships between variables of cooking oil fume exposure and the severity of COPD according to GOLD criteria ( $N=632$ )

\begin{tabular}{|c|c|c|c|c|c|c|c|}
\hline \multirow[t]{2}{*}{ Variables } & \multirow{2}{*}{$\begin{array}{l}\text { No COPD }(N=336) \\
\text { N }(\%)\end{array}$} & \multicolumn{3}{|c|}{ Mild COPD $(N=223)$} & \multicolumn{3}{|c|}{ Moderate COPD $(N=73)$} \\
\hline & & $\bar{N}(\%)$ & COR $(95 \% \mathrm{Cl})$ & AOR $(95 \% \mathrm{Cl})^{*}$ & $\bar{N}(\%)$ & COR $(95 \% \mathrm{Cl})$ & AOR $(95 \% \mathrm{Cl})^{*}$ \\
\hline \multicolumn{8}{|l|}{ Smoking status } \\
\hline Non-smoker & $108(32.1)$ & 66 (29.6) & 1 & 1 & $11(15.1)$ & 1 & 1 \\
\hline Second-hand smoker & $228(67.9)$ & $157(70.4)$ & $1.13(0.78-1.63)$ & $1.03(0.70-1.52)$ & $62(84.9)$ & $2.67(1.35-5.27)$ & $2.98(1.47-6.01)$ \\
\hline \multicolumn{8}{|l|}{ Cooked in the kitchen } \\
\hline No & $24(7.1)$ & $12(5.4)$ & 1 & 1 & $2(2.7)$ & 1 & 1 \\
\hline Daily & $312(92.9)$ & $211(94.6)$ & $1.35(0.66-2.76)$ & $1.07(0.51-2.27)$ & $71(97.3)$ & $2.73(0.63-11.82)$ & $2.04(0.46-9.18)$ \\
\hline \multicolumn{8}{|l|}{ Excluding no cook } \\
\hline \multicolumn{8}{|l|}{ Age stared cooking } \\
\hline$>22$ years & $139(44.6)$ & $104(49.3)$ & 1 & 1 & $33(46.5)$ & 1 & 1 \\
\hline$\leq 22$ years & $173(55.5)$ & $107(50.7)$ & $0.83(0.58-1.17)$ & $0.69(0.47-1.00)$ & $38(53.5)$ & $0.93(0.55-1.55)$ & $0.87(0.49-1.52)$ \\
\hline \multicolumn{8}{|l|}{ Meals per week ${ }^{\mathrm{a}}$} \\
\hline $1-13$ & $85(27.2)$ & $45(21.3)$ & 1 & 1 & $13(18.3)$ & 1 & 1 \\
\hline $14-20$ & $100(32.1)$ & $76(36.0)$ & $1.44(0.90-2.29)$ & $1.39(0.86-2.25)$ & $15(21.1)$ & $0.98(0.44-2.18)$ & $0.85(0.37-1.94)$ \\
\hline $21-31$ & $127(40.7)$ & $90(42.7)$ & $1.34(0.85-2.10)$ & $1.23(0.77-1.98)$ & $43(60.6)$ & $2.21(1.12-4.36)$ & $1.63(0.80-3.33)$ \\
\hline \multicolumn{8}{|l|}{ Fuel used for cooking ${ }^{b}$} \\
\hline Gas/electric stove & $194(62.4)$ & $125(59.2)$ & 1 & 1 & $33(46.5)$ & 1 & 1 \\
\hline Biomass fuels & $74(23.8)$ & $52(24.6)$ & $1.09(0.72-1.66)$ & $0.89(0.54-1.49)$ & $21(29.6)$ & $1.67(0.91-3.07)$ & $0.87(0.41-1.85)$ \\
\hline Coal & $43(13.8)$ & $34(16.1)$ & $1.23(0.74-2.03)$ & $1.12(0.64-1.98)$ & $17(23.9)$ & $2.32(1.19-4.55)$ & $1.36(0.63-2.97)$ \\
\hline \multicolumn{8}{|c|}{ Windows in the home kitchen ${ }^{c}$} \\
\hline No & $22(7.2)$ & $12(5.9)$ & 1 & 1 & $4(5.7)$ & 1 & 1 \\
\hline$\geq 1$ & $285(92.8)$ & $192(94.1)$ & $1.24(0.60-2.56)$ & $1.31(0.62-2.77)$ & $66(94.3)$ & $1.27(0.43-3.82)$ & $1.67(0.54-5.20)$ \\
\hline \multicolumn{8}{|c|}{ Ventilation in the home kitchen ${ }^{d}$} \\
\hline Poor & $14(4.5)$ & $8(3.8)$ & 1 & 1 & $7(9.9)$ & 1 & 1 \\
\hline Good & $297(95.5)$ & $201(96.2)$ & $1.18(0.49-2.88)$ & $1.18(0.48-2.94)$ & $64(91.0)$ & $0.43(0.17-1.11)$ & $0.49(0.19-1.30)$ \\
\hline \multicolumn{8}{|l|}{ Installed fume extractor ${ }^{\mathrm{e}}$} \\
\hline No & $131(42.7)$ & $99(48.3)$ & 1 & 1 & $39(54.9)$ & 1 & 1 \\
\hline Yes & $176(57.3)$ & $106(51.7)$ & $0.80(0.56-1.14)$ & $0.85(0.55-1.29)$ & $32(45.1)$ & $0.61(0.36-1.03)$ & $1.01(0.55-1.86)$ \\
\hline \multicolumn{8}{|c|}{ Eye irritation during cooking ${ }^{f}$} \\
\hline No & $277(97.5)$ & $174(93.6)$ & 1 & 1 & $60(93.8)$ & 1 & 1 \\
\hline Yes & $7(2.5)$ & $12(6.5)$ & $2.73(1.05-7.06)$ & $3.02(1.14-8.00)$ & $4(6.3)$ & $2.64(0.75-9.30)$ & $1.96(0.54-7.17)$ \\
\hline \multicolumn{8}{|c|}{ Smokiness during cooking ${ }^{9}$} \\
\hline No & $267(93.7)$ & $171(91.9)$ & 1 & 1 & $52(81.3)$ & 1 & 1 \\
\hline Yes & $18(6.3)$ & $15(8.1)$ & $1.30(0.64-2.65)$ & $1.22(0.59-2.53)$ & $12(18.8)$ & $3.42(1.56-7.53)$ & $2.89(1.28-6.55)$ \\
\hline
\end{tabular}

$A O R$ adjusted OR, COPD chronic obstructive pulmonary diseases, COR crude OR, GOLD Global Initiative for Chronic Obstructive Lung Disease, OR odds ratio ${ }^{*}$ Adjusting for age, second hand smoke status, height, education level, burning incense and tea consumption

${ }^{\mathrm{a}}$ Missing data, $n=10(0,10,0) .{ }^{\mathrm{b}}$ Missing data, $n=11(1,10,0) .{ }^{\mathrm{C}}$ Missing data, $n=13(5,7,1) .{ }^{\mathrm{d}}$ Missing data, $n=3(1,2,0) .{ }^{\mathrm{e}}$ Missing data, $n=11(5,6,0) .{ }^{\mathrm{f}}$ Missing data, $n=60$ $(28,25,7)$. ${ }^{9}$ Missing data, $n=59(27,25,7)$

and genotoxicity but also inflammatory or irritant reactions in the airways. With regard to genotoxicity, for example, in-vitro and human studies have reported 8-hydroxy-2'deoxyguanosine causes dose-dependent increases in the levels of B[a]P 7,8-diol 9,10-epoxide N2-deoxyguanosineDNA adducts and oxidative DNA damage when exposure to COF or fume extracts [28-30].
In addition to having carcinogenic effects, exposure to chemicals from fume condensates may cause airway injuries and inflammatory responses through their effect on oxidant and antioxidant imbalances and innate immunity impairment [31-33]. Wu et al., investigating the chemical trans-trans-2,4-decadienal (t-t-2,4-DDE) detected in peanut oil fumes, found glutathione (GSH) 
Table 4 Relationships between variables of cooking oil fume exposure and pulmonary function (absolute FEV 1 level and FEV $1 / F V C$ (\%)) in multiple linear regression models

\begin{tabular}{|c|c|c|c|c|c|c|c|}
\hline \multirow[t]{2}{*}{ Variables } & \multirow[b]{2}{*}{$\mathrm{N}$} & \multirow[b]{2}{*}{$\begin{array}{l}\text { FEV1 }(\mathrm{L}) \\
(\text { mean } \pm \mathrm{SD})\end{array}$} & \multicolumn{2}{|c|}{ Adjusted analysis ${ }^{*}$} & \multirow[b]{2}{*}{$\begin{array}{l}\text { FEV1/FVC (\%) } \\
(\text { mean } \pm \text { SD) }\end{array}$} & \multicolumn{2}{|c|}{ Adjusted analysis* } \\
\hline & & & $\overline{\beta(S E)}$ & $p$-value & & $\overline{\beta(S E)}$ & $p$-value \\
\hline \multicolumn{8}{|l|}{ Smoking status } \\
\hline Non-smoker & 187 & $1.57 \pm 0.44$ & 1 & - & $87.24 \pm 13.00$ & 1 & - \\
\hline Second-hand smoker & 450 & $1.57 \pm 0.47$ & $-0.054(0.033)$ & 0.108 & $81.73 \pm 15.05$ & $-5.71(1.27)$ & $<0.0001$ \\
\hline \multicolumn{8}{|l|}{ Cooked in the kitchen } \\
\hline No & 39 & $1.70 \pm 0.46$ & 1 & - & $88.15 \pm 11.97$ & 1 & - \\
\hline Yes & 598 & $1.56 \pm 0.46$ & $-0.07(0.06)$ & 0.270 & $83.03 \pm 14.80$ & $-4.84(2.45)$ & 0.048 \\
\hline \multicolumn{8}{|l|}{ Excluding no cook } \\
\hline \multicolumn{8}{|l|}{ Age stared cooking } \\
\hline$>22$ years & 278 & $1.58 \pm 0.46$ & 1 & - & $82.03 \pm 14.56$ & 1 & - \\
\hline$\leq 22$ years & 320 & $1.55 \pm 0.45$ & $0.029(0.032)$ & 0.374 & $83.90 \pm 14.98$ & $1.46(1.23)$ & 0.235 \\
\hline \multicolumn{8}{|l|}{ Meals per week } \\
\hline $1-13$ & 145 & $1.69 \pm 0.47$ & 1 & - & $84.98 \pm 14.08$ & 1 & - \\
\hline $14-20$ & 192 & $1.58 \pm 0.43$ & $-0.027(0.042)$ & 0.525 & $84.81 \pm 12.84$ & $0.15(1.61)$ & 0.927 \\
\hline $21-31$ & 261 & $1.47 \pm 0.46$ & $-0.065(0.041)$ & $0.111^{\dagger}$ & $80.64 \pm 16.19$ & $-3.35(1.55)$ & $0.031^{+}$ \\
\hline \multicolumn{8}{|l|}{ Fuel used for cooking } \\
\hline Gas/electric stove & 354 & $1.69 \pm 0.44$ & 1 & - & $83.46 \pm 13.17$ & 1 & - \\
\hline Biomass fuels & 147 & $1.38 \pm 0.41$ & $-0.036(0.044)$ & 0.414 & $82.56 \pm 16.19$ & $1.18(1.70)$ & 0.487 \\
\hline Coal & 96 & $1.34 \pm 0.42$ & $-0.103(0.048)$ & $0.034^{\ddagger}$ & $82.06 \pm 18.06$ & $0.60(1.86)$ & 0.746 \\
\hline \multicolumn{8}{|c|}{ Windows in the home kitchen } \\
\hline No & 38 & $1.59 \pm 0.57$ & 1 & - & $81.68 \pm 16.11$ & 1 & - \\
\hline$\geq 1$ & 546 & $1.56 \pm 0.45$ & $-0.122(0.064)$ & 0.056 & $83.27 \pm 14.75$ & $0.75(2.46)$ & 0.760 \\
\hline \multicolumn{8}{|c|}{ Ventilation in the home kitchen } \\
\hline Poor & 29 & $1.42 \pm 0.51$ & 1 & - & $76.28 \pm 16.45$ & 1 & - \\
\hline Good & 566 & $1.57 \pm 0.46$ & $0.103(0.073)$ & 0.156 & $83.41 \pm 14.67$ & $5.89(2.77)$ & 0.034 \\
\hline \multicolumn{8}{|l|}{ Installed fume extractor } \\
\hline No & 271 & $1.41 \pm 0.42$ & 1 & - & $81.91 \pm 15.90$ & 1 & - \\
\hline Yes & 316 & $1.69 \pm 0.45$ & $0.041(0.037)$ & 0.261 & $83.89 \pm 13.87$ & $0.43(1.41)$ & 0.759 \\
\hline \multicolumn{8}{|c|}{ Eye irritation during cooking } \\
\hline No & 515 & $1.59 \pm 0.45$ & 1 & - & $82.92 \pm 14.43$ & 1 & - \\
\hline Yes & 23 & $1.32 \pm 0.62$ & $-0.158(0.082)$ & 0.055 & $76.15 \pm 22.16$ & $-5.34(3.12)$ & 0.088 \\
\hline \multicolumn{8}{|c|}{ Smokiness during cooking } \\
\hline No & 493 & $1.59 \pm 0.44$ & 1 & - & $83.33 \pm 13.96$ & 1 & - \\
\hline Yes & 46 & $1.41 \pm 0.57$ & $-0.137(0.059)$ & 0.021 & $75.07 \pm 21.08$ & $-7.67(2.24)$ & 0.001 \\
\hline
\end{tabular}

*Adjusting for age, height, second-hand smoking status, education level, burning incense and tea consumption

${ }^{+}$Trend test from 1 to $13,14-20$, to $21-31$ meals per week in FEV1: $p=0.100$; in FEV1/FVE(\%): $p=0.016$

${ }^{\ddagger}$ Trend test from gas/electric stove, biomass fuels, to coal in FEV1: $p=0.037$

content as well as the activities of antioxidative enzymes such as GSH reductase, GSH peroxidase and GSH Stransferase were reduced by the methanolic extract of oil fumes [34]. That study found that t-t-2,4-DDE produced superoxide anion, hydrogen peroxide, and hydroxyl radicals in a phosphate buffer ( $\mathrm{pH} 7.4$ ) and found that it induced intracellular reactive oxidative stress (ROS) in
A-549 cells. Another study reported similar findings in oil fumes created by heating three common commercial cooking oils (soybean oil, sunflower oil, and lard) [35]. In one recent published paper, Peng and her colleagues, studying COF levels resulting from different kinds of cooking methods and uses of cooking oils, found that use of palm oil or rapeseed oil could reduce COF 
exposure, especially for long-chain aldehydes such as hexanal and t,t-2,4-DDE [36]. Tung et al. also reported that COF exposure induced cytokine expression (TGF $\beta 1$ ) and oxidative stress in CL3 lung epithelial cells [37]. These findings suggest COF-induced inflammatory response in the airways may lead to respiratory symptoms or lung function impairment and contribute to development of chronic bronchitis.

Many epidemiological studies have reported an association between COF exposure and lung cancer risk [5, 15-18]. One recent meta-analysis summarized 13 articles including three population-based case-control studies and ten hospital-based case-control studies in nonsmoking Chinese women, totaling 3596 women with lung cancer and 6082 healthy controls [27]. That meta-analysis found the pooled estimates of risk ratio in fixed effects model and random effects model to be $1.74 \quad(95 \%$ CI $=1.57-1.94)$ and $2.11 \quad(95 \%$ CI =1.54-2.89), respectively. Very few studies, however, have investigated the effect of COF exposure on nonmalignant respiratory diseases [34, 38, 39]. Most of the studies that did study this effect focused on indoor smoke exposure when biomass fuels were used (e.g., wood, excrement, straw, etc.) while cooking [38, 39]. Dennis et al, conducting a case-control study in Colombia, found the use of wood for cooking to be significantly associated with the development of obstructive airways disease $(\mathrm{OR}=3.43 ; p<0.001)$ [38]. Another population survey has also found biomass fuel use (especially wood) to be an important deteriorating factor for pulmonary function values, including $\mathrm{FEV}_{1}, \mathrm{FEV}_{1} \%$, peak expiratory flow rate (PEFR) and mid-flow rate (defined as the forced expiratory flow (FEF) from $25 \%$ to $75 \%$ of the vital capacity) in females [39]. In one study of professional chefs, Svendsen and colleagues reported that female kitchen workers had higher prevalence rates of dyspnea (relative risk $(R R)=4.1$; 95\% $\mathrm{CI}=2.7-6.3)$, serious dyspnea $(\mathrm{RR}=2.9 ; 95 \% \mathrm{CI}=$ 1.5-5.7)), and respiratory symptoms related to work $(\mathrm{RR}=4.3 ; 95 \% \mathrm{CI}=2.7-6.7)$ compared to their female controls, suggesting that there is an association between exposure to cooking fumes and the development of respiratory diseases other than cancer in kitchen workers.

Cigarette smoking is a well-known major risk factor for both malignant and nonmalignant respiratory diseases. Indeed, in a previous study, we found that women who smoked and women who had been exposed to a lifetime of SHS were 24.81 times (95\% CI: 5.78-106.38) and 3.65 times (95\% CI: 1.19-11.26) more likely to have chronic bronchitis than those who had not been exposed to SHS [7]. In the current study, we also found that SHS exposure to be major contributor the development of chronic nonmalignant respiratory diseases among nonsmoking women. The adverse effects of COF exposure in this study were probably not as strong as those of
SHS exposure (Table 2; Table 3). The reasons for this difference may be that (1) the concentrations of respiratory irritants maybe lower in COF chemicals than SHS chemicals or (2) the information about COF exposure we collected maybe not as accurate as that of SHS exposure. We found reproducibility of some COF exposure variables queried on our survey to have moderate agreement; thus, any misclassification would probably be random and cause an underestimation of the significance.

This study has several limitations. As mentioned above, data on COF exposure, including cooking activities, different kinds of oils, and ventilation systems etc., were collected by questionnaire. This would give rise to the possibility of recall bias, though that bias would likely be random and result in a null effect. For our outcomes of interest, the only objective measurement in this study was the pulmonary function test. Thus, there is a likelihood for some information bias in our subjective measurements. Another limitation is the occurrence of competing risk with cancer, since the previous studies have associated COF exposure with various cancers, especially lung cancer [19]. The age range at which malignant respiratory diseases occur is younger than the ages that nonmalignant respiratory usually occur. This might lead to a systematic underestimation of the prevalence of COPD in this population-based study. Another limitation is that we had no data on the participant's past disease status, because our insurance health dataset had only the diagnostic codes recorded for that year (1999). This might lead to a random misclassification of outcome, though this possibility is reduced by fact that we also used ATS criteria to confirm original diagnoses reported by their physicians. Because this is an observational study and chronic bronchitis/COPD is a multifactorial disease, other unmeasured confounders could have potentially affected our findings.

\section{Conclusion}

In conclusion, the frequency of meals cooked per week and kitchen smokiness during cooking between the ages of 20- and 40-years-old are important contributing factors for chronic bronchitis and impaired lung function in nonsmoking Taiwanese women. Good kitchen ventilation may reduce the lung function impairment. Future studies may want to explore optimal design for kitchen ventilators to decrease the aggravation of COPD caused by $\mathrm{COF}$ exposure.

\section{Additional file}

Additional file 1: Table S1. Distributions of demographic characteristics categorized by study cases and controls among non-smoking women $(N=644)$. Table S2. The distribution across our study subjects with different health statuses. Table S3. Relationships between major variable of cooking oil 
fume exposure and other covariates in non-cook women and the risk of chronic bronchitis among non-smoking women $(N=644)$. Table S4. Relationships between cooking and ventilation conditions in home kitchens between 20 and 40 years old and the severity of COPD according to GOLD criteria ( $N=632$ ). Figure S1. Study area. (DOCX 64 kb)

\section{Acknowledgements}

We are grateful to all the interviewers who worked in this study.

\section{Funding}

This work was supported by Kaohsiung Medical University (KMU-TP104A00 and KMU-TP104A07), the Ministry of Science and Technology (MOST 104-2314-B-037012-MY2), Taiwan's National Health Research Institutes (NHRI-EX102-10209PI), and Taiwan Ministry of Health and Welfare, none of which had any role in study design, data collection and analysis, decision to publish, or preparation of the manuscript.

\section{Availability of data and materials}

The datasets supporting the conclusions of this article are included within the article. The datasets generated during the current study are available in the Research Center for Environmental Medicine, Kaohsiung Medical University, Kaohsiung, Taiwan, at https://envmed.kmu.edu.tw/index.php/en-GB/datarepository/168-exposure-to-cooking-oil-fumes-and-chronic-bronchitis-innonsmoking-women-aged-40-years-and-over-a-health-care-based-study.

\section{Authors' contributions}

MTW had full access to all of the data in the study and takes responsibility for the integrity of the data and the accuracy of the data analysis. Study concept and design: CHC, CFW, IWC and MTW Acquisition of data: CHC, IWC and CFW Analysis and interpretation of data: CHC, CFW, IWC and MTW Drafting of the manuscript: $\mathrm{CHC}$ and CFW Critical revision of the manuscript for important intellectual content: CHC, CFW, IWC and MTW Statistical analysis: CFW and MTW Study supervision: MTW All authors read and approved the final manuscript.

\section{Ethics approval and consent to participate}

Participants were informed of the details of the study and inform consent was obtained and documented in the questionnaire form before specimen collection. This study was approved by IRB at Kaohsiung Medical University. All participants provided signed informed consent. The use of this dataset has been approved by Taiwan's Kao-Ping District National Health Insurance Bureau, a branch of Taiwan's National Health Insurance Program.

\section{Consent for publication}

Not applicable.

\section{Competing interests}

The authors declare that they have no competing interests.

\section{Publisher's Note}

Springer Nature remains neutral with regard to jurisdictional claims in published maps and institutional affiliations.

\footnotetext{
Author details

'Department of Internal Medicine, Kaohsiung Municipal Hsiao-Kang Hospital, Kaohsiung Medical University, Kaohsiung, Taiwan. ${ }^{2}$ Department of Public Health, College of Health Sciences, Kaohsiung Medical University, Kaohsiung, Taiwan. ${ }^{3}$ Research Center for Environmental Medicine, Kaohsiung Medical University, Kaohsiung, Taiwan. ${ }^{4}$ Division of Pulmonary and Critical Care Medicine, Department of Internal Medicine, Kaohsiung Medical University Hospital, Kaohsiung, Taiwan. ${ }^{5}$ Faculty of Medicine, College of Medicine, Kaohsiung Medical University Hospital, Kaohsiung, Taiwan. ${ }^{6}$ Department of Family Medicine, Kaohsiung Medical University Hospital, Kaohsiung Medical University, Kaohsiung, Taiwan.
}

Received: 22 March 2017 Accepted: 1 February 2018

Published online: 13 February 2018

\section{References}

1. World Health Organization, The 10 leading causes of death in the world, 2000 and 2012, Updated May 2014. http://www.who.int/mediacentre/ factsheets/fs310/en/ Accessed on 19 Dec 2017.

2. Cheng SL, Chan MC, Wang CC, Lin CH, Wang HC, Hsu JY, Hang LW, Chang CJ, Perng DW, Yu CJ. COPD in Taiwan: a National Epidemiology Survey. Int J Chron Obstruct Pulmon Dis. 2015;10:2459-67.

3. Department of Statistics, Ministry of Health and Welfare, Taiwan, 2011 National Health Insurance Annual Statistical (Chinese version). https://www. mohw.gov.tw/np-131-2.html/. Accessed 19 Dec 2017.

4. Assad NA, Balmes J, Mehta S, Cheema U, Sood A. Chronic obstructive pulmonary disease secondary to household air pollution. Semin Respir Crit Care Med. 2015;36:408-21.

5. Ko YC, Cheng LS, Lee CH, Huang JJ, Huang MS, Kao EL, Wang HZ, Lin HJ. Chinese food cooking and lung cancer in women nonsmokers. Am J Epidemiol. 2000;151:140-7.

6. Gram IT, Austin H, Stalsberg H. Cigarette smoking and the incidence of cervical intraepithelial neoplasia, grade III, and cancer of the cervix uteri. Am J Epidemiol. 1992;135:341-6.

7. Wu CF, Feng NH, Chong IW, Wu KY, Lee CH, Hwang JJ, Huang CT, Lee CY, Chou ST, Christiani DC, Wu MT. Second-hand smoke and chronic bronchitis in Taiwanese women: a health-care based study. BMC Public Health. 2010; 10:44.

8. Chiang TA, Pei-Fen W, Ying LS, Wang LF, Ko YC. Mutagenicity and aromatic amine content of fumes from heated cooking oils produced in Taiwan. Food Chem Toxicol. 1999:37:125-34.

9. Li S, Pan D, Wang G. Analysis of polycyclic aromatic hydrocarbons in cooking oil fumes. Arch Environ Health. 1994;49:119-22.

10. Chiang TA, Wu PF, Ko YC. Prevention of exposure to mutagenic fumes produced by hot cooking oil in Taiwanese kitchens. Environ Mol Mutagen. 1998;31:92-6.

11. Hudnell HK, Otto DA, House DE, Mølhave L. Exposure of humans to a volatile organic mixture. II. Sensory. Arch Environ Health. 1992;47:31-8.

12. Chiang TA, Wu PF, Wang LF, Lee $\mathrm{H}$, Lee $\mathrm{CH}$, Ko YC. Mutagenicity and polycyclic aromatic hydrocarbon content of fumes from heated cooking oils produced in Taiwan. Mutat Res. 1997;381:157-61.

13. Shields PG, Xu GX, Blot WJ, Fraumeni JF Jr, Trivers GE, Pellizzari ED, Qu YH, Gao YT, Harris CC. Mutagens from heated Chinese and U.S. cooking oils. J Natl Cancer Inst 1995;87:836-841.

14. Zhu X, Wang K, Zhu J, Koga M. Analysis of cooking oil fumes by ultraviolet spectrometry and gas chromatography-mass spectrometry. J Agric Food Chem. 2001:49:4790-4.

15. Wang TJ, Zhou BS, Shi JP. Lung cancer in nonsmoking Chinese women: a case-control study. Lung Cancer. 1996;14(Suppl 1):S93-8.

16. Zhong L, Goldberg MS, Gao YT, Jin F. Lung cancer and indoor air pollution arising from Chinese-style cooking among nonsmoking women living in shanghai, China. Epidemiology. 1999;10:488-94.

17. Metayer C, Wang Z, Kleinerman RA, Wang L, Brenner AV, Cui H, Cao J, Lubin $\mathrm{JH}$. Cooking oil fumes and risk of lung cancer in women in rural Gansu, China. Lung Cancer. 2002;35:111-7.

18. Wen Cheng $Y$, Lee H. Environmental exposure and lung cancer among nonsmokers: an example of Taiwanese female lung cancer. J Environ Sc Health C Environ Carcinog Ecotoxicol Rev. 2003;21:1-28.

19. Wu MT, Lee LH, Ho CK, Wu SC, Lin LY, Cheng BH, Liu CL, Yang CY, Tsai HT, Wu TN. Environmental exposure to cooking oil fumes and cervical intraepithelial neoplasm. Environ Res. 2004;94:25-32.

20. Cheng SH, Chiang TL. The effect of universal health insurance on health care utilization in Taiwan. Results from a natural experiment. JAMA. 1997; 278:89-93.

21. Lee CH, Yang SF, Peng CY, Li RN, Chen YC, Chan TF, Tsai EM, Kuo FC, Huang JJ, Tsai HT, Hung YH, Huang HL, Tsai S. Wu MT. the precancerous effect of emitted cooking oil fumes on precursor lesions of cervical cancer. Int J Cancer. 2010;127:932-41.

22. Viera AJ, Garrett JM. Understanding interobserver agreement: the kappa statistic. Fam Med. 2005:37:360-3.

23. Standards for the diagnosis and care of patients with chronic obstructive pulmonary disease. American Thoracic Society. Am J Respir Crit Care Med. 1995;152:S77-S121. 
24. Baldwin ED, Cournand A, Richards DW Jr. Pulmonary insufficiency; physiological classification, clinical methods of analysis, standard values in normal subjects. Medicine. 1948;27:243-78.

25. Berglund E, Birath G, Bjure J, Grimby G, Kjellmer I, Sandqvist L, Soderholm B. Spirometric studies in normal subjects. I. Forced expirograms in subjects between 7 and 70 years of age. Acta Med Scand. 1963;173:185-92.

26. Rabe KF, Hurd S, Anzueto A, Barnes PJ, Buist SA, Calverley P, Fukuchi Y, Jenkins C, Rodriguez-Roisin R, van Weel C, Zielinski J. Global initiative for chronic obstructive lung disease. Global strategy for the diagnosis, management, and prevention of chronic obstructive pulmonary disease: GOLD executive summary. Am J Respir Crit Care Med. 2007:176:532-55.

27. Xue Y, Jiang Y, Jin S, Li Y. Association between cooking oil fume exposure and lung cancer among Chinese nonsmoking women: a meta-analysis. Onco Targets Ther. 2016;9:2987-92.

28. Yang SC, Jenq SN, Kang ZC, Lee H. Identification of benzo[a]pyrene 7,8-diol 9,10-epoxide N2-deoxyguanosine in human lung adenocarcinoma cells exposed to cooking oil fumes from frying fish under domestic conditions. Chem Res Toxicol. 2000;13:1046-50.

29. Pan $\mathrm{CH}$, Chan CC, Wu KY. Effects on Chinese restaurant workers of exposure to cooking oil fumes: a cautionary note on urinary 8-hydroxy-20-deoxyguanosine. Cancer Epidemiol Biomark Prev. 2008;17:3351-7.

30. Cherng SH, Huang KH, Yang SC, Wu TC, Yang JL, Lee H. Human 8-oxoguanine DNA glycosylase 1 mRNA expression as an oxidative stress exposure biomarker of cooking oil fumes. J Toxicol Environ Health A. 2002;65:265-78.

31. Olloquequi J, Silva OR. Biomass smoke as a risk factor for chronic obstructive pulmonary disease: effects on innate immunity. Innate Immun. 2016;22:373-81.

32. Norback D, Bjornsson E, Janson C, Widstrom J, Boman G. Asthmatic symptoms and volatile organic compounds, formaldehyde, and carbon dioxide in dwellings. Occup Environ Med. 1995;52:388-95.

33. Simpson FG, Belfield PW, Cooke NJ. Chronic airflow limitation after inhalation of overheated cooking oil fumes. Postgrad Med J. 1985;61:1001-2.

34. Wu SC, Yen GC. Effects of cooking oil fumes on the genotoxicity and oxidative stress in human lung carcinoma (A-549) cells. Toxicol in Vitro. 2004;18:571-80

35. Dung CH, Wu SC, Yen GC. Genotoxicity and oxidative stress of the mutagenic compounds formed in fumes of heated soybean oil, sunflower oil and lard. Toxicol in Vitro. 2006;20:439-47.

36. Peng CY, Lan CH, Lin PC, Kuo YC. Effects of cooking method, cooking oil, and food type on aldehyde emissions in cooking oil fumes. J Hazard Mater. 2017;324:160-7.

37. Tung YH, Ko JL, Liang YF, Yin L, Pu Y, Lin PP. Cooking oil fume induced cytokine expression and oxidative stress in human lung epithelial cells. Environ Res. 2001;87:47-54.

38. Dennis RJ, Maldonado D, Norman S, Baena E, Martinez G. Woodsmoke exposure and risk for obstructive airways disease among women. Chest. 1996:109:115-9.

39. Saha A, Rao NM, Kulkarni PK, Majumdar PK, Saiyed HN. Pulmonary function and fuel use: a population survey. Respir Res. 2005;6:127.

\section{Submit your next manuscript to BioMed Central and we will help you at every step:}

- We accept pre-submission inquiries

- Our selector tool helps you to find the most relevant journal

- We provide round the clock customer support

- Convenient online submission

- Thorough peer review

- Inclusion in PubMed and all major indexing services

- Maximum visibility for your research

Submit your manuscript at www.biomedcentral.com/submit

) Biomed Central 